\title{
Study on the Evolution Characteristics of International Nickel Ores Trade from Perspective of Complex Networks
}

\author{
Yun Ding ${ }^{1}$ \\ ${ }^{1}$ School of Humanities and Economic Management, China University of Geosciences, Beijing, 100083.China
}

\begin{abstract}
In order to analyze the evolution characteristics of nickel ores international trade structure, this paper builds up the network model by adopting the complex network theory. Based on the International trade data of nickel ores from 2007 to 2018, we analyze the overall pattern of trade network, core country identification and the power-law distribution analysis. According to the research, it is found that China and the United States have a strong influence in the international nickel ores trading network. The more nickel producing counties participated in this trade and the international nickel ore market commenced to globalization.
\end{abstract}

\section{Introduction}

Nickel is an excellent widely-used metal due to its high temperature resistance, strong ductility and oxidation resistance. Now nickel is mainly used in the manufacture of stainless steel, high nickel alloy steel and alloy structural steel[1]. Nickel ore is a very important non-ferrous metal for the basic industry of a country, especially in the military industry. With the promotion of economic globalization, the international nickel ore trade is also growing and expanding. There are many international nickel ore trading countries, and the trading relationships among them constitute an intricate trading network. By using the method of complex networks, it can find out the internal evolution rules of the international nickel ores trade.

In recent years, the method of complex networks is widely used in many fields, such as financial area, medical area, IT area, transportation area and international trade area. In the field of international trade, some scholars have studied the evolution laws of the international trade of the resources, such as iron ore, copper ore, natural gas, oil, and bauxite, and further provide policy advices[2-4]. The predecessors have not analyzed the evolution of the international nickel ore trading by using the complex network method. Therefore, this paper can provide policy reference for the international nickel ore trade market, and has important theoretical and practical significance.

\section{Methodology and data}

\subsection{Data Source}

The statistical data comes from the UN comtrade database, which records the relevant information of international nickel ores trade from 2007 to 2018. In the database, the code number is 260400 which stands for nickel ores and concentrates. The data of exporting countries, importing countries and trade volume was screened and all the relevant data was selected as of April 1st, 2019.

\subsection{Network Model Construction}

The paper defines countries which participate in the nickel trade each year as the nodes, the trade relationship between countries as the conjunctional edges and the volume of nickel trade as the weights of the edges. By using these factors, the directed weighted complex network of the international nickel trade in that year is established.

The network is represented by the adjacent square matrix $\mathrm{T}(\mathrm{t})$, as the following equation (1).

$$
T(t)=\left[\begin{array}{ccc}
w_{1,1}(t) & \cdots & w_{1, j}(t) \\
\vdots & \ddots & \vdots \\
w_{1, i}(t) & \cdots & w_{i, j}(t)
\end{array}\right]
$$

In the equation, $w_{i, j}(t)$ represents the volume of nickel ore exported by country $i$ to country $j$ in $t$ year. In The complex network of international nickel ores trade is as the following Figure 1. 


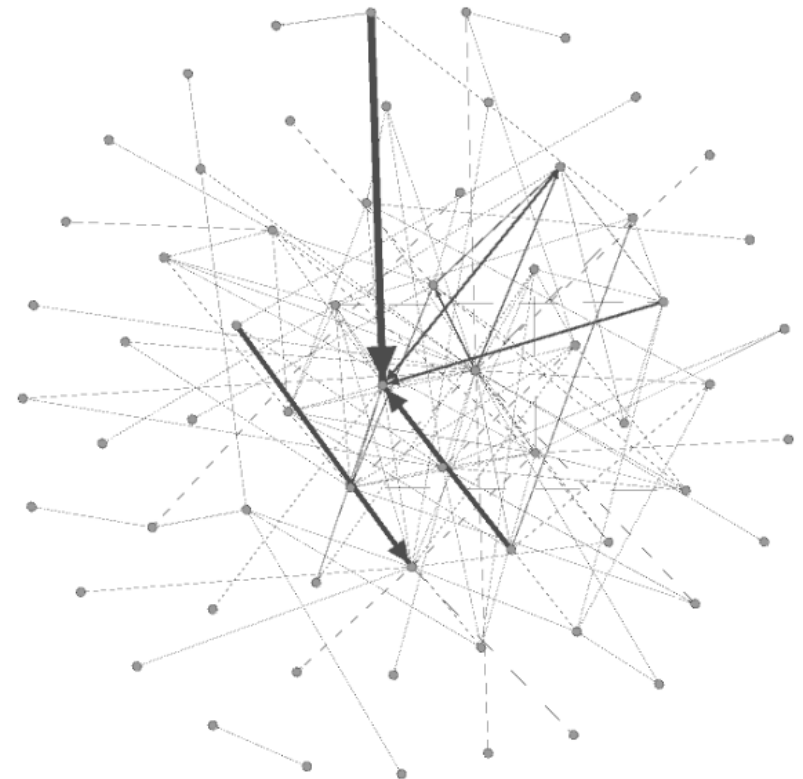

Figure 1. The complex network of international nickel ores trade in the year of 2018

In Figure 1, the nodes stand for the countries participating in the international nickel ores trade of the year 2018, and the edges represent the nickel trading relationships between counties. As the Figure 1 shown, the international nickel ores trade network is complex, and different countries due to its contributions is located in different positions in the network.

\subsection{Indicators Analysis}

\section{Definition 1. Degree}

Degree is defined as the number of links incident upon a node[5]. Accordingly, degree is a count of the number of ties directed to the node. In complex network, the more ties one node is connected with, the more influential one it will be, and the higher this node's centrality is. The degree $k_{i}$ is calculated as follow:

$$
k_{i}=\sum_{i, j e N} d_{i j}
$$

Where $d_{i j}$ is a binary data whose value is 1 and 0 . When there is a tie between node $i$ and node $j$, the value is 1 . On the contrary, value 0 represents no ties between node $i$ and node $j . N$ represents the vertex of all nodes.

The average degree value is a reflection of the overall network connection.

\section{Definition 2. Weighted Degree}

Weighted degree of a node is a reflection of the weight of the edge connected to the node[5]. It represents the total trading volume of a country. In the complex network, the node has the bigger weighted degree, which means the bigger trading volume and the more important status. The weighted degree $\mathrm{Si}$ is calculated as follow:

$$
\mathrm{S}_{i}(\mathrm{t})=\sum_{j=1}^{N(t)} w_{i j}(t)
$$

\author{
Where $w_{i j}(t)$ is the weighted from node $\mathrm{i}$ to node $\mathrm{j}$. \\ ${ }^{N(t)}$ stands for the total number of countries in the year of \\ t.
}

\section{Definition 3. Network Density}

Network density refers to the closeness of the connections between various nodes. In the real world, it is to show the closeness of connections between countries as a whole. The closeness of trade links between countries is higher, which means the regional grouping tends to strong. The network density $\mathrm{C}$ is calculated as follow:

$$
\mathrm{C}=2 \mathrm{~m} / \mathrm{n}(\mathrm{n}-1)
$$

In the equation, $\mathrm{n}$ stands for the number of nodes, and $\mathrm{m}$ represents the real number of the relationships in the network. The value of $\mathrm{C}$ ranges from 0 to 1 . When the value of $\mathrm{C}$ closes to 1 , the network density is high and the connections among nodes are closely. While, when the value of $\mathrm{C}$ nears to 0 , the connections among nodes are distributed.

\section{Results and discussions}

Based on the above data and the complex network indicators, UCINET6.0 software is used to calculate the degree, average degree, weighted degree and network density in the international nickel ores trading network.

\subsection{Overall pattern of trade network analysis}

The average degree value is a reflection of the overall network connection. The evolution characteristics of the average degree value of the nodes are shown in Figure 1. From 2007 to 2018, the average degree value of the nodes in the network displays an upward trend year by year, indicating that the number of trading partnerships between trading countries is gradually increasing. The international nickel trade has become closer and more connected. In Figure 2, the average degree value of nodes in the network declined from the year of 2007 to 2009. Because the world-scale financial crisis broke out globally, and the global economy fell into a downturn. The sluggish economic environment affected the nickel trade situation during these period. After the year of 2010 , the international nickel trade situation gradually returned to increase.

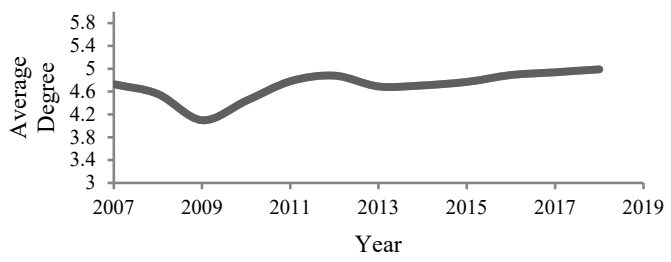

Figure 2. The evolution curve of the average degree of nodes in the network

The network density is a reflection of the closeness of 
connections among countries. As Table 1 shown, from the year of 2007 to 2018 , the range of the network density of international nickel ore trade relations is from 0.035 to 0.044 . During the year of 2007 to 2009, the network density experienced significant fluctuations. In 2007, the world-scale financial crisis led to the unstable nickel ores trade and affected the connections among countries. After the financial crisis, the connection among countries was going to closely and the network density was going to increase.

Table1. Network density of international nickel ore trade relations

\begin{tabular}{ccccccc}
\hline Year & 2007 & 2008 & 2009 & 2010 & 2011 & 2012 \\
\hline Density & 0.039 & 0.031 & 0.037 & 0.038 & 0.036 & 0.035 \\
\hline & & & & & & \\
\hline Year & 2013 & 2014 & 2015 & 2016 & 2017 & 2018 \\
\hline Density & 0.035 & 0.037 & 0.037 & 0.039 & 0.041 & 0.044 \\
\hline
\end{tabular}

In summary, by analyzing the evolutionary characteristics of the average degree of nodes and network density in the international nickel trade network, we can find that the number of countries participating in international nickel trading is increasing, the scale of international nickel trade is constantly growing, and more and more countries have established nickel trade relationships.

\subsection{Core country identification}

The status of a country can be determined by its location in the network. The importance of each country's status in the network depends on the number of its trading partners and its contribution to the economic development of the trading volume. The number of trading partners in the network is the degree of the node. The contribution to the economic development of the trading is the trading volume, and the trading volume can be expressed by the weighted degree of the node in the network. By using the equation 2 and 3, the degree value and the weighted degree value of each country can be calculated, as following Table 2 and Table 3.

Table 2. The ranking of trading country's degree

\begin{tabular}{ccccc}
\hline Ranking & 2012 & 2014 & 2016 & 2018 \\
\hline 1 & China & China & China & China \\
2 & USA & USA & South & USA \\
& South & South & Zimbabwe & Canada \\
3 & Africa & Africa & Germany & Zimbabwe \\
4 & Germany & Zimbabwe & German \\
5 & Finland & Canada & Indonesia & Germany
\end{tabular}

As shown in Table 2,the top three countries are China, USA, and South Africa. China is always the country with the highest rankings, that is, China has the most trading partners due to its huge of consumption. The rankings of USA and South Africa are following China, and they also have many trading partners; while the other countries, such as the Germany, Zimbabwe, Finland, Canada, and Indonesia also have a certain number of trading partners.
Table 3. The ranking of trading country's weighted degree

\begin{tabular}{ccccc}
\hline Ranking & 2012 & 2014 & 2016 & 2018 \\
\hline 1 & USA & China & China & China \\
2 & China & USA & South & USA \\
3 & South Africa & Germany & USA & Canada \\
4 & Germany & South & Zimbabwe & Finland \\
5 & Belgium & Finland & Germany & Germany \\
\hline
\end{tabular}

According to Table 3, the countries with higher rankings include China, USA, and South Africa, which mean these countries have the largest volume of nickel trade. The weighted degree of China has been ranked first since 2014; While, some countries such as Germany, Zimbabwe, Canada and Finland, rank lower than China and other countries, which indicates that these countries still have a certain influence in the network.

\subsection{Power-law distribution analysis}

In the real world, many complex networks have power-law distribution characteristics, such as the financial system network and so on. In these networks, the degree distribution of nodes is uneven. The degrees of only some nodes are bigger, while the degrees of most nodes are smaller. From the year of 2007 to 2018, the fitting function of the nodes' degree distribution is around 0.76 in the international nickel ore trade network. As Figure1shown, the degree distribution of nodes has the power-law distribution characteristics. The power-law distribution network has a feature of preferential attachment. In the international nickel ore trade network, the counties which late participate in prefer to corporate with the core counties. The core counties such as China and the United States will have more and more trading partners and the strong influence in the nickel ore trade network.

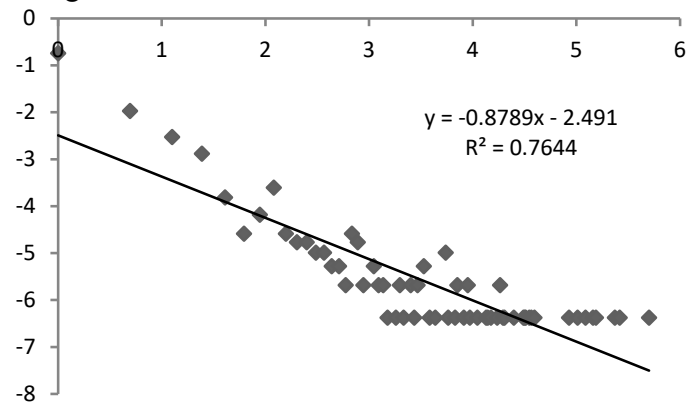

Figure3. The fitting function of the nodes' degree distribution in the year of 2018

\section{Conclusions}

This paper studies evolution characteristics of international nickel ores trade by using the relevant theoretical knowledge of complex networks. It finds that the number of countries participating in the international nickel trade has increased, the trade relationships between countries have gradually increased, and the volume of trade has also increased. The overall scale of the international nickel trade network has continued to grow, that means prosperous development trend of the nickel ore trade. In the international nickel ores trade 
network, China occupies the most important position due to the largest number of trading partners and the volume of nickel trade.

\section{References}

1. Z. Liang, A.S. Feng, Global Nickel Resources Development and Utilization Status and Supply and Demand Analysis, Mineral Protection and Utilization, 2 64-69 (2016)

2. D. Di, H.Z. An, International Copper Ore Trade Pattern Based on Complex Network Theory, Economic Geography, 36 94-101 (2016)
3. C.Y. Shi, X.Y. Gao, Study on the evolution characteristics of international bauxite trade from the perspective of complex network, China Mining Magazine, Lecture Notes on network analysis, 27 57-62 (2018)

4. X.Q. Hao, H.Z. An, Study on the evolution characteristics of international iron ore trade from the perspective of complex network, Economic Geography, 33 92-97 (2013)

5. D. Matthias, Structural analysis of complex networks,(2010) 